\title{
Chia sẻ tri thức và hành vi làm việc đổi mới của nhân viên trong ngành hàng không
}

\section{Knowledge sharing and innovative work behaviour in the aviation industry}

\author{
Đoàn Bảo Sơn ${ }^{1 *}$ \\ ${ }^{1}$ Học viện Hàng không Việt Nam, Việt Nam \\ *Tác giả liên hệ, Email: sondb@vaa.edu.vn
}

THÔNG TIN

DOI: $10.46223 /$ HCMCOUJS. econ.vi.17.3.1871.2022

Ngày nhận: 04/05/2021

Ngày nhận lại: 10/06/2021

Duyệt đăng: 15/06/2021

\section{TÓM TẮT}

Dựa vào các lý thuyết hiện tại, nghiên cứu này phát triển mô hình về mối quan hệ giữa chia sẻ tri thức và hành vi làm việc đổi mới (IWB) của nhân viên trong ngành hàng không với vai trò thúc đẩy của niềm tin (TRU), hệ thống phần thưởng của tổ chức (REW), sự hỗ trợ của quản lý (MAS). Tác giả kiểm chứng các giả thuyết nghiên cứu bằng mô hình cấu trúc tuyến tính (SEM), với số liệu điều tra từ 280 mẫu trả lời của các đáp viên là nhân viên đang làm việc tại Cảng Hàng không Quốc tế Tân Sơn Nhất. Dữ liệu được thu thập bằng bảng hỏi và sử dụng phương pháp lấy mẫu thuận tiện. Kết quả thực nghiệm cho thấy TRU, REW và MAS có ảnh hưởng tích cực đến chia sẻ tri thức hiện $(\mathrm{KNO})$ và chia sẻ tri thức ẩn (TKNO). Đồng thời, IWB được thúc đẩy bởi KNO và TKNO. Nghiên cứu này đề xuất với các nhà lãnh đạo trong ngành hàng không một số hàm ý quản trị khuyến khích nhân viên tăng cường chia sẻ tri thức và làm việc đổi mới.

\section{ABSTRACT}

Tù khóa:

chia sẻ tri thức; hành vi làm việc đổi mới; niềm tin; phần thưởng của tổ chức; sự hỗ trợ của quản lý

Keywords:

knowledge sharing; innovative work behavior; trust; reward; management support
Adapted from current literatures, this study develops the model of the relationship between knowledge sharing and employees' Innovative Work Behavior (IWB) in the aviation industry with the promoting of trust (TRU), Organizational Rewards system (REW), Management Support (MAS). The author tested the research hypotheses using a Structural Equation Modeling (SEM), with survey data from 280 respondents who were employees working at Tan Son Nhat International Airport. Structured questionnaires are designed to collect data using convenience sampling. The empirical studys demonstrated that TRU, REW and MAS positively impacting on explicit knowledge sharing (KNO) and tacit knowledge sharing (TKNO). At the same time, IWB is driven by KNO and TKNO. Research results contribute some practical implications to stimulate the strength of knowledge sharing and innovative work in the aviation industry. 


\section{Giới thiệu}

Tri thức là nguồn lực chủ yếu và tạo ra giá trị của tổ chức, là một chức năng của khả năng tích lũy và sử dụng tri thức (Hsu \& Sabherwal, 2012; Zhou \& Li, 2012). Chia sẻ và chuyển giao tri thức sẽ hình thành một nguồn tri thức liên quan đến năng lực, do đó góp phần tạo ra tài sản và nâng cao hiệu quả của tổ chức (Z. Wang, Wang, \& Liang, 2014). Trong môi trường cạnh tranh cao, chia sẻ tri thức là nhân tố quan trọng mang đến sự thành công cho doanh nghiệp (Grant, 1996). Khuyến khích nhân viên chia sẻ tri thức hữu ích trong tổ chức có thể duy trì và làm tăng lợi thế cạnh tranh của tổ chức đó (Barney, 1991; Grant, 1996; Liu \& Phillips, 2011). Đối với các công ty hàng không, tri thức của nhân viên là rất cần thiết để giữ chân và thu hút khách hàng mới, nhằm đáp ứng nhu cầu ngày càng cao và cải thiện các dịch vụ cung cấp cho họ. Để tạo ra trải nghiệm khách hàng mới và độc đáo, gần đây các nhà quản lý trong ngành hàng không đã bắt đầu quan tâm đến các thực hành chia sẻ tri thức (Hu, Horng, \& Sun, 2009).

Có thể thấy rằng, thách thức lớn trong việc thúc đẩy hành vi đổi mới trong công việc là sự sẵn sàng của cá nhân trong việc chia sẻ tri thức giữa các đồng nghiệp với nhau. Có hai vấn đề liên quan đến khía cạnh này. Một là nhận thức cá nhân, dựa trên năng lực bản thân và kỳ vọng kết quả (Hsu, Ju, Yen, \& Chang, 2007; S. Wang \& Noe, 2010). Hai là tác động xã hội dựa trên niềm tin và các tiêu chuẩn chủ quan (Bock, Zmud, Kim, \& Lee, 2005; Hsu \& ctg., 2007; S. Wang \& Noe, 2010). Điều tra các nhận thức cá nhân và tác động của môi trường xã hội đối với hành vi làm việc đổi mới thông qua chia sẻ tri thức sẽ giúp người quản lý thúc đẩy chia sẻ tri thức trong các đơn vị hoặc tổ chức nhằm thúc đẩy sự sáng tạo, đổi mới của nhân viên (Bock \& ctg., 2005; Radaelli, Lettieri, Mura, \& Spiller, 2014).

Các công ty hàng không muốn thành công thì cần cung cấp các dịch vụ mới, chất lượng cao và cải tiến để tạo ra trải nghiệm độc đáo cho khách hàng. Để đạt được mục tiêu này, các nhà quản lý cần có chính sách để đảm bảo rằng nhân viên của họ thường xuyên chia sẻ tri thức với những người khác để tạo ra các dịch vụ giá trị gia tăng. Bên cạnh việc học tập của tổ chức và hiệu quả đổi mới, việc chia sẻ kinh nghiệm của bản thân sẽ giúp mọi người trong tổ chức hiểu được nhu cầu, kỳ vọng, mong muốn và sở thích của hành khách để có thể đóng góp tích cực vào sự phát triển dịch vụ mới và hành vi làm việc đổi mới (Afsar, Safdar, Dost, \& Ali, 2017).

Trong thời gian vừa qua, các doanh nghiệp trong ngành hàng không Việt Nam đã triển khai nhiều chương trình, chính sách nhằm nâng cao năng lực đổi mới và tạo ra lợi thế cạnh tranh, góp phần thúc đẩy sự phát triển kinh tế, văn hóa, xã hội của đất nước cũng như nâng cao chất lượng phục vụ hành khách. Tuy nhiên, dự báo sắp tới hoạt động vận tải hàng không trong nước và quốc tế sẽ gặp nhiều khó khăn, thách thức. Do đó, việc nghiên cứu các nhân tố thúc đẩy sự đổi mới và nâng cao hiệu quả làm việc của đội ngũ nhân viên trong ngành hàng không là thật sự cần thiết. Hơn nữa, tác động của niềm tin, phần thưởng của tổ chức và sự hỗ trợ của quản lý đối với chia sẻ tri thức và hành vi làm việc đổi mới của nhân viên trong ngành hàng không tại Việt Nam thông qua điều tra thực nghiệm và kiểm định bằng mô hình cấu trúc tuyến tính (SEM) là một khoảng trống nghiên cứu. Nghiên cứu này sẽ lắp đầy khoảng trống đó bằng cách phân tích các tiền đề và kết quả của chia sẻ tri thức trong bối cảnh Tổng công ty Hàng không Việt Nam. Kết quả của nghiên cứu này sẽ giúp các nhà lãnh đạo, quản lý trong ngành hàng không có các chính sách nhằm thúc đẩy hành vi chia sẻ tri thức và nâng cao chất lượng đổi mới.

\section{Cơ sở lý thuyết và phát triển các giả thuyết}

\subsection{Các nhân tố thúc đẩy chia sẻ tri thức}

\section{Niềm tin (Trust)}

Nhân tố làm cải thiện các mối quan hệ tương tác và thúc đẩy chia sẻ tri thức thành công 
giữa các nhân viên trong tổ chức là niềm tin (Chow \& Chan, 2008). Các sáng kiến chia sẻ tri thức trong tổ chức phụ thuộc vào niềm tin. Khi niềm tin được thiết lập, nhân viên sẵn sàng cho phép người khác truy nhập các nguồn lực của họ (nghĩa là tài sản trí tuệ, vật chất và tình cảm của chính họ). Nói cách khác, những cá nhân tham gia vào các mối tương tác dựa trên niềm tin sẽ sẵn sàng chia sẻ nguồn lực hoặc tri thức chuyên môn của họ với nhau bởi vì cảm giác tin tưởng khiến họ giảm bớt sự lo sợ (Tsai \& Ghoshal, 1998). Niềm tin sẽ góp phần nâng cao chất lượng chia sẻ tri thức (Zaqout \& Abbas, 2012) và là nền tảng cho sự gắn kết quan hệ giữa các bên, ngăn chặn các hành vi cơ hội và góp phần trao đổi tri thức miễn phí (Inkpen \& Tsang, 2005). Sự sẵn sàng chia sẻ tri thức với người khác sẽ tăng lên khi các nhân viên tin rằng điều đó sẽ tăng cường mối quan hệ, mở rộng phạm vi liên kết cũng như thúc đẩy hợp tác và cộng tác cho công việc trong tương lai (Javaid, Soroya, \& Mahmood, 2020). Ngoài ra, Foos, Schum, và Rothenberg (2006) cho rằng niềm tin giữa các cá nhân sẽ thúc đẩy chia sẻ tri thức ẩn. Dựa trên các luận điểm này, nghiên cứu đề xuất các giả thuyết $\mathrm{H} 1$ và $\mathrm{H} 2$ như sau:

\section{H1: Niềm tin có tác động tích cực đến chia sẻ tri thức hiện}

H2: Niềm tin có tác động tích cực đến chia sẻ tri thức ẩn

\section{Phần thưởng (Reward)}

Phần thưởng có thể là tiền tệ và phi tiền tệ. Phần thưởng bằng tiền có thể là tiền thưởng, sự khuyến khích. Phần thưởng phi tiền tệ có thể là giấy chứng nhận, sự công nhận của công chúng, sự đánh giá cao. Nhiều học giả đã công nhận tầm quan trọng của phần thưởng trong chia sẻ tri thức (Javaid \& ctg., 2020).

Phần thưởng của tổ chức có ảnh hưởng tích cực trong việc thúc đẩy nhân viên thực hiện các hành vi công việc khác nhau và nếu nhân viên có được nhiều phần thưởng hơn đối với việc chia sẻ tri thức thì mức độ sẵn sàng chia sẻ tri thức của họ sẽ càng tăng lên (Cabrera, Collins, \& Salgado, 2006; Huang, Davison, \& Gu, 2011). Khi nhân viên nhận được phần thưởng kinh tế cho việc trao đổi tri thức của họ, họ cảm thấy có nhiều động lực hơn để chia sẻ tri thức (Hau, Kim, Lee, \& Kim, 2013). Kết quả nghiên cứu của Cheng, Ho, và Lau (2009) đã cho thấy tầm quan trọng của việc xác lập các hệ thống khuyến khích đầy đủ và sự hiểu biết về kỳ vọng đối với việc chia sẻ tri thức của một cá nhân.

Dựa vào các lập luận trên, nghiên cứu này đề xuất các giả thuyết $\mathrm{H} 3$ và $\mathrm{H} 4$ như sau:

H3: Phần thuơơng của tổ chức có tác động tích cực đến chia sẻ tri thức hiện

H4: Phần thưởng của tổ chức có tác động tích cực đến chia sẻ tri ẩn

\section{Sự hỗ trọ̣ của quản lý (Management support)}

Sự hỗ trợ của quản lý đóng một vai trò quan trọng đối với việc khuyến khích các hành vi chia sẻ tri thức (Chen \& Cheng, 2012; S. Wang \& Noe, 2010). Hỗ trợ quản lý đề cập đến sự hỗ trợ mà quản lý cấp cao và cấp trung có các biện pháp để tăng cường các hành vi và quy trình chia sẻ tri thức trong toàn tổ chức. Hỗ trợ quản lý được định nghĩa là cách mà các nhà quản lý truyền cảm hứng cho nhân viên chia sẻ tri thức và hỗ trợ các hoạt động khuyến khích chia sẻ tri thức (J. C. Lee, Shiue, \& Chen, 2016 ; Z. Wang \& Wang, 2012). Mishra và Pandey (2019) cho rằng các phong cách lãnh đạo khác nhau có ảnh hưởng tích cực đến việc chia sẻ tri thức. Lãnh đạo hiệu quả có tiềm năng mạnh mẽ để nâng cao mức độ và chất lượng của việc chia sẻ tri thức bằng cách tạo ra văn hóa thân thiện với tri thức, cung cấp các mục tiêu chung và tầm nhìn rõ ràng, cải thiện các mối quan hệ dựa trên lòng tin, sử dụng các động cơ thúc đẩy và loại bỏ các rào cản như thiếu giao tiếp hoặc hiểu lầm lẫn nhau (Amayah, 2013). 
Anantatmula (2008) cho rằng lãnh đạo, quản lý đóng một vai trò quan trọng đối với văn hóa chia sẻ tri thức trong một tổ chức, bởi vì trong việc chia sẻ tri thức, trách nhiệm không chỉ thuộc về nhân viên mà còn là của các nhà quản lý cấp cao, những người cần thiết lập một môi trường thúc đẩy sự chia sẻ tri thức. Sự hỗ trợ của quản lý là tiền đề cho việc chia sẻ tri thức trong các bối cảnh nghiên cứu khác nhau, vì sự hồ trợ của quản lý có khả năng ảnh hưởng đến cam kết của nhân viên, từ đó cải thiện chất lượng và mức độ thực tiễn chia sẻ tri thức (Ali, Paris, \& Gunasekaran, 2019).

Dựa vào các lý thuyết hiện tại, nghiên cứu đề xuất các giả thuyết $\mathrm{H} 5$ và $\mathrm{H} 6$ như sau:

H5: Sự hỗ trọ của quản lý có tác động tích cực đến chia sẻ tri thức hiện

H6: Sụ hỗ trợ của quản lý có tác động tích cực đến chia sẻ tri thức ẩn

\subsection{Chia sẻ tri thức và hành vi làm việc đổi mới của nhân viên}

Đổi mới là quá trình mà qua đó giá trị kinh tế hoặc xã hội tạo ra từ tri thức - thông qua việc sáng tạo, phổ biến và chuyển đổi tri thức để tạo ra các sản phẩm, quy trình cải tiến mới hoặc được cải thiện đáng kể được xã hội sử dụng (Raykov, 2014). Do đó, đổi mới là một chiến lược cạnh tranh thiết yếu và lợi thế cho bất kỳ sự tồn tại của tổ chức nào và làm cho tổ chức đó có sự khác biệt. Hơn nữa, nó thu hút nhiều khách hàng hơn do thực tế là những điều mới luôn hấp dẫn. Đặc biệt ngành dịch vụ cần đổi mới liên tục để giữ chân khách hàng (Akram, Lei, Haider, \& Hussain, 2018).

Tri thức là nguồn lực tổ chức quan trọng nhất, cho phép tạo ra các kết quả tổ chức mới bao gồm cả sự đổi mới (Kamasak \& Bulutlar, 2010). Hơn nữa, chia sẻ tri thức giúp mọi người nhanh chóng mở rộng phạm vi tri thức cá nhân và tăng khả năng giải quyết vấn đề và khối lượng công việc (Hu \& ctg., 2009). Các lý thuyết hiện tại hỗ trợ cho mối quan hệ giữa chia sẻ tri thức và đổi mới (Akram \& ctg., 2018). Alhady, Idris, Sawal, Azmi, và Zakaria (2011) cho rằng tổ chức hỗ trợ nhân viên của mình đóng góp tri thức với mong muốn sẽ tạo ra những ý tưởng mới, tốt hơn và thúc đẩy các cơ hội mới, từ đó cho phép các hoạt động đổi mới tổ chức. Khi cá nhân thu thập tri thức từ người khác, họ cải thiện năng lực đổi mới của mình (Radaelli \& ctg., 2014). Nhân viên có thể giải quyết được những vấn đề một cách hiệu quả thông qua những những ý tưởng sáng tạo, đổi mới nhờ vào việc chia sẻ tri thức với đồng nghiệp (K. J. Lee, 2016; Nonaka, 1994). Tiền đề cho sự kết hợp và chuyển từ tri thức hiện có sang tri thức mới là chia sẻ tri thức, kết quả sẽ thúc đẩy thực hiện các ý tưởng sáng tạo, tác động tích cực đến hiệu quả làm việc (Yun \& Lee, 2017). Chia sẻ tri thức hiện sẽ nâng cao hiệu quả học tập, làm thay đổi cấu trúc tri thức của nhân viên. Bên cạnh đó, các nhân viên trong tổ chức có thể có được và sử dụng các kỹ năng, kinh nghiệm và phương pháp đặc biệt để làm việc tốt hơn nhờ vào đổi mới nhận thức và chia sẻ tri thức ẩn (Z. Wang \& ctg., 2014). Do đó, tác giả đề xuất rằng có mối quan hệ tích cực giữa chia sẻ tri thức hiện và chia sẻ tri thức ẩn với hành vi làm việc đổi mới của các cá nhân bằng các giả thuyết $\mathrm{H} 7$ và $\mathrm{H} 8$ như sau:

H7: Chia sẻ tri thức hiện có ảnh hương tích cục đến hành vi làm việc đổi mới H8: Chia sẻ tri thức ẩn có ảnh hưởng tích cực đến hành vi làm việc đổi mới 


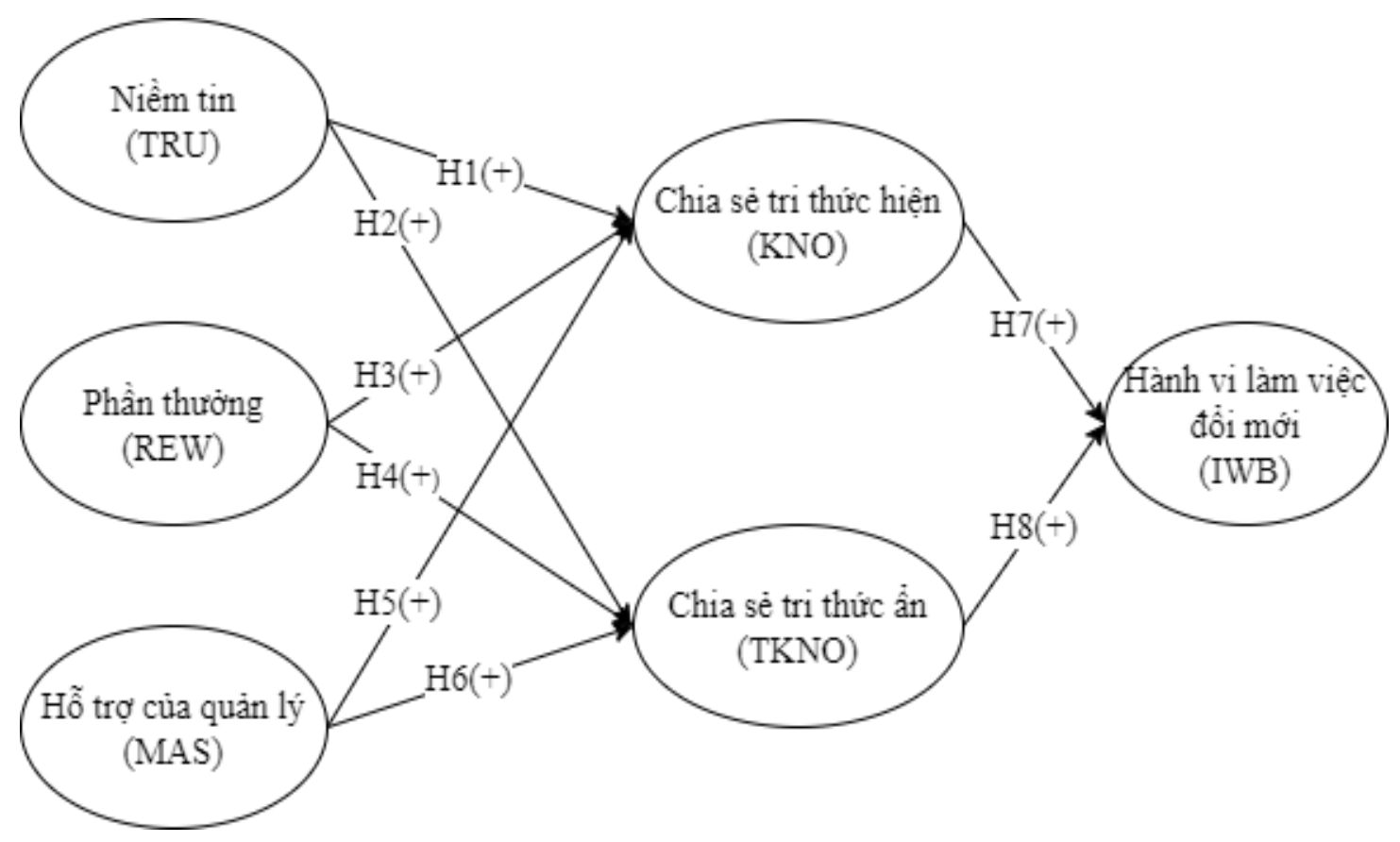

Hình 1. Mô hình nghiên cứu đề xuất

Nguồn: Tác giả

\section{Phương pháp nghiên cứu}

\subsection{Chọn mẫu và thu thập dĩ liệu}

Nghiên cứu này sử dụng bảng câu hỏi được thiết kế sẵn và phương pháp chọn mẫu thuận tiện để thu thập dữ liệu. Các đáp viên của khảo sát là nhân viên của Tổng Công ty Hàng không Việt Nam (Vietnam Airlines) đang công tác tại Cảng Hàng không Quốc tế Tân Sơn Nhất. Đối với mô hình SEM, để đạt được độ tin cậy cần thiết thì cỡ mẫu càng lớn càng tốt, nhưng tối thiểu phải bằng 05 lần số biến quan sát (Hair, Black, Babin, \& Anderson, 2014; Kline, 2015). Trong mô hình nghiên cứu này có 06 biến tiềm ẩn với 33 biến quan sát, do đó số lượng mẫu ít nhất phải bằng 200 . Tác giả đã tiến hành khảo sát trong thời gian từ tháng 01 đến tháng 02 năm 2021 bằng cách gửi trực tiếp và trực tuyến 350 bảng câu hỏi đến nhân viên của Vietnam Airlines. Sau khi loại bỏ các trả lời không đầy đủ, tác giả thu được 328 phiếu (đạt tỷ lệ hồi đáp $93.7 \%$ ), trong đó có 280 mẫu là chuẩn để tiến hành phân tích dữ liệu.

\subsection{Thang đo}

Các biến quan sát trong bài nghiên cứu này được đo lường theo thang đo Likert 5 mức độ, trong đó " 1 " là "hoàn toàn không đồng ý" và " 5 " là "hoàn toàn đồng ý." Tác giả phát triển các thang đo dựa vào các công trình nghiên cứu trước trong cùng lĩnh vực. Thang đo niềm tin (ký hiệu là TRU) gồm 03 biến được tham khảo từ Javaid và cộng sự (2020). Phần thưởng của tổ chức (ký hiệu là REW) gồm 04 biến quan sát được phát triển theo Bock và Kim (2002). Dựa theo kết quả nghiên cứu của Ali và cộng sự (2019), sự hỗ trợ của quản lý (ký hiệu là MAS) được đo lường bởi 04 biến. Đối với các cấu trúc chia sẻ tri thức, chia sẻ tri thức hiện (ký hiệu là $\mathrm{KNO}$ ) gồm 06 biến và chia sẻ tri thức ẩn (ký hiệu là $T K N O)$ gồm 07 biến được tham khảo từ Reychav và Weisberg (2010) và $Z$. Wang và Wang (2012). Cuối cùng, nghiên cứu sử dụng 09 biến để đo lường hành vi làm việc đổi mới (IWB) (Akram \& ctg., 2018; Janssen, 2000). Các kết quả trong Bảng 1 cho thấy hệ số Cronbach's Alpha của tất cả các biến tiềm ẩn đều lớn hơn 0.8 nên thang đo có độ tin cậy là rất tốt (Kline, 2015). Cùng với đó là hệ số tương quan biến tổng của tất cả các biến quan sát đều lớn hơn 0.6. Vì vậy, tất cả các biến quan sát đều được chấp nhận và sẽ được sử dụng trong phân tích nhân tố. 


\section{Bảng 1}

Kết quả phân tích Cronbach's Alpha

\begin{tabular}{|c|c|c|}
\hline Nhân tố & Hệ số Cronbach's Alpha & Tương quan biến tổng \\
\hline 1. Niềm tin (TRU) & 0.934 & \\
\hline TRU-1 & & 0.869 \\
\hline TRU-2 & & 0.857 \\
\hline TRU-3 & & 0.881 \\
\hline 2. Phần thưởng của tổ chức (REW) & 0.874 & \\
\hline REW-1 & & 0.761 \\
\hline REW-2 & & 0.766 \\
\hline REW-3 & & 0.674 \\
\hline REW-4 & & 0.728 \\
\hline 3. Hỗ trợ của quản lý (MAS) & 0.897 & \\
\hline MAS-1 & & 0.800 \\
\hline MAS-2 & & 0.805 \\
\hline MAS-3 & & 0.790 \\
\hline MAS-4 & & 0.689 \\
\hline 4. Chia sẻ tri thức hiện (KNO) & 0.923 & \\
\hline KNO-1 & & 0.784 \\
\hline KNO-2 & & 0.808 \\
\hline KNO-3 & & 0.770 \\
\hline KNO-4 & & 0.787 \\
\hline KNO-5 & & 0.779 \\
\hline KNO-6 & & 0.743 \\
\hline 5. Chia sẻ tri thức ẩn (TKNO) & 0.945 & \\
\hline TKNO-1 & & 0.748 \\
\hline TKNO-2 & & 0.837 \\
\hline TKNO-3 & & 0.865 \\
\hline TKNO-4 & & 0.810 \\
\hline TKNO-5 & & 0.817 \\
\hline TKNO-6 & & 0.795 \\
\hline TKNO-7 & & 0.831 \\
\hline 6. Hành vi làm việc đổi mới (IWB) & 0.938 & \\
\hline IWB-1 & & 0.771 \\
\hline IWB-2 & & 0.725 \\
\hline
\end{tabular}




\begin{tabular}{|c|c|c|}
\hline Nhân tố & Hệ số Cronbach's Alpha & Tương quan biến tổng \\
\hline IWB-3 & & 0.774 \\
\hline IWB-4 & & 0.782 \\
\hline IWB-5 & & 0.703 \\
\hline IWB-6 & & 0.790 \\
\hline IWB-7 & & 0.804 \\
\hline IWB-8 & & 0.791 \\
\hline IWB-9 & & 0.739 \\
\hline
\end{tabular}

Nguồn: Tác giả

\subsection{Phân tích dĩu liệu}

Theo khuyến nghị của Anderson và Gerbing (1988), nghiên cứu này phân tích dữ liệu theo phương pháp hai bước bằng phần mềm thống kê SPSS 22 và AMOS 24. Đầu tiên, mô hình đo lường được thiết lập bằng phân tích nhân tố khẳng định (CFA) để kiểm định tính giá trị hội tụ và tính giá trị phân biệt. Tiếp theo, tác giả phân tích đường dẫn và ước lượng các hệ số tương quan đường dẫn bằng mô hình SEM.

\section{Kết quả nghiên cứu}

\section{1. Đặc điểm nhân hẩu học của mẫu nghiên cúu}

Đặc điểm nhân khẩu học về giới tính, độ tuổi, trình độ học vấn và vị trí công tác của 280 đáp viên được mô tả cụ thể trong Bảng 2.

\section{Bảng 2}

Bảng mô tả mẫu nghiên cứu

\begin{tabular}{|c|c|c|}
\hline Đặc điểm & Số lượng & Tỉ lệ phần trăm (\%) \\
\hline $\begin{array}{l}\text { Tuổi: } \\
\text { - Nhỏ nhất: } 23 \\
\text { - Lớn nhất: } 59 \\
\text { - Trung bình: } 36.84\end{array}$ & & \\
\hline $\begin{array}{l}\text { Giới tính: } \\
\text { - Nữ } \\
\text { - Nam }\end{array}$ & $\begin{array}{l}135 \\
145\end{array}$ & $\begin{array}{l}48.2 \\
51.8\end{array}$ \\
\hline $\begin{array}{l}\text { Trình độ học vấn: } \\
\text { - Cao đằng } \\
\text { - Đại học } \\
\text { - Thạc sĩ }\end{array}$ & $\begin{array}{c}23 \\
194 \\
63 \\
\end{array}$ & $\begin{array}{c}8.2 \\
69.3 \\
22.5 \\
\end{array}$ \\
\hline $\begin{array}{l}\text { Vị trí công tác: } \\
\text { - Nhân viên } \\
\text { - Lãnh đạo }\end{array}$ & $\begin{array}{c}242 \\
38\end{array}$ & $\begin{array}{l}86.4 \\
13.6\end{array}$ \\
\hline \multicolumn{3}{|c|}{ Số mẫu N = 280} \\
\hline
\end{tabular}

Nguồn: Tác giả tổng hợp từ EVIEWS 


\subsection{Phân tích mô hình đo lường}

\subsubsection{Sụ phù hợp của mô hình đo luòng (Model Fit)}

Sau khi phân tích nhân tố khám phá (EFA), 04 biến quan sát (IWB-6, IWB-7, IWB-8 và IWB-9) trong cấu trúc hành vi làm việc đổi mới (IWB) đã bị loại bỏ do chúng có tải chéo (Hair \& ctg., 2014). Như vậy, từ 06 biến tiềm ẩn và 33 biến quan sát ban đầu, mô hình còn 29 biến quan sát.

Trong bài báo này, tác giả tham chiếu theo các khuyến nghị của Hair và cộng sự (2014, tr. 584) để đánh giá độ phù hợp của mô hình đo lường, với trường hợp cụ thể số quan sát trong mỗi nhóm $\mathrm{N}>250$ và số biến quan sát $12<\mathrm{m}<30$. Dựa vào các kết quả thống kê, mô hình đo lường này có chỉ số $\mathrm{CMIN} / \mathrm{df}=1.902$ nhỏ hơn 3 và giá trị $\mathrm{P}$-value $=0.000$ không lớn hơn 0.05 (Hair \& ctg., 2014). Ngoài ra, chỉ số phù hợp so sánh (CFI) bằng 0.951 , chỉ số Tucker-Lewis (TLI) bằng 0.945 đều vượt mức ngưỡng 0.92 ; cùng với sai số ước lượng căn quân phương (RMSEA) bằng 0.057 nhỏ hơn 0.07 và chỉ số thống kê phần dư chuẩn hóa căn quân phương (SRMR) bằng 0.049 nhỏ hơn giá trị cho phép 0.080 (Hair \& ctg., 2014). Các bằng chứng này dẫn đến kết luận mô hình đo lường là hoàn toàn phù hợp (Hair \& ctg., 2014).

\subsubsection{Tính giá trị (Validity) của mô hình}

Tính giá trị của mô hình đo lường được tác giả đánh giá bằng hai kiểm định, đó là tính giá trị hội tụ và tính giá trị phân biệt (Anderson \& Gerbing, 1988). Trong đó, tính giá trị hội tụ được kiểm tra bằng cách đánh giá độ tin cậy tổng hợp $(\mathrm{CR})$ của các cấu trúc trong mô hình và phương sai trích trung bình (AVE) của các cấu trúc đó. Các số liệu thực nghiệm trong Bảng 3 cho thấy độ tin cậy $\mathrm{CR}$ của tất cả 06 biến tiềm ẩn nằm trong khoảng từ 0.877 (REW) đến $0.945(\mathrm{TKNO})$, nghĩa là đều đạt trên giá trị cắt 0.7 (Hair \& ctg., 2014) và giá trị AVE của cả 06 biến tiềm ẩn này cũng đều vượt mức ngưỡng 0.5 (Hair \& ctg., 2014), chúng nằm trong khoảng từ 0.642 (REW) đến 0.831 (TRU). Do đó, có thể kết luận mô hình đo lường đạt yêu cầu về sự hội tụ. Đồng thời, bình phương hệ số tương quan lớn nhất (MSV) của các cấu trúc đều nhỏ hơn phương sai trích (AVE). Vì thế, có thể kết luận mô hình đo lường cũng đạt yêu cầu về sự phân biệt (Hair \& ctg., 2014). Như vậy, tính giá trị hội tụ và tính giá trị phân biệt của mô hình đo lường đã được kiểm định bằng các số liệu điều tra thực nghiệm.

\section{Bảng 3}

Độ tin cậy và tính giá trị của mô hình CFA

\begin{tabular}{|l|c|c|c|c|c|c|c|c|c|}
\hline & CR & AVE & MSV & TKNO & KNO & IWB & MAS & REW & TRU \\
\hline TKNO & 0.945 & 0.711 & 0.561 & $\mathbf{0 . 8 4 3}$ & & & & & \\
\hline KNO & 0.923 & 0.667 & 0.561 & $0.749 * * *$ & $\mathbf{0 . 8 1 7}$ & & & & \\
\hline IWB & 0.904 & 0.657 & 0.258 & $0.488^{* * *}$ & $0.486^{* * *}$ & $\mathbf{0 . 8 1 1}$ & & & \\
\hline MAS & 0.897 & 0.687 & 0.396 & $0.550^{* * *}$ & $0.528^{* * *}$ & $0.348^{* * *}$ & $\mathbf{0 . 8 2 9}$ & & \\
\hline REW & 0.877 & 0.642 & 0.307 & $0.476^{* * *}$ & $0.523^{* * *}$ & $0.321 * * *$ & $0.335 * * *$ & $\mathbf{0 . 8 0 1}$ & \\
\hline TRU & 0.937 & 0.831 & 0.515 & $0.718^{* * *}$ & $0.700^{* * *}$ & $0.508^{* * *}$ & $0.629 * * *$ & $0.554 * * *$ & $\mathbf{0 . 9 1 2}$ \\
\hline
\end{tabular}

Nguồn: Tác giả 


\subsection{Phân tích mô hình đường dẫn}

\subsubsection{Kiểm định sụ phù hợp của mô hình cấu trúc}

Trong nghiên cứu này, sự phù hợp của mô hình cấu trúc cũng được đánh giá dựa trên đề xuất của Hair và cộng sự (2014), với các chỉ số đánh giá tương tự như mô hình đo lường. Các kết quả phân tích cho thấy rằng chỉ số $\mathrm{CMIN} / \mathrm{df}=2.032$ nhỏ hơn $3 ; \mathrm{P}$-value $=0.000$ không lớn hơn $0.05 ; \mathrm{CFI}=0.944$ lớn hơn $0.92 ; \mathrm{TLI}=0.937$ lớn hơn $0.92 ; \mathrm{RMSEA}=0.061$ nhỏ hơn 0.07 và $\mathrm{SRMR}=0.0721$ nhỏ hơn 0.08 . Do đó, có thể kết luận rằng mô hình cấu trúc là phù hợp (Hair \& ctg., 2014).

\subsubsection{Kiểm định các giả thuyết nghiên cứu}

Bảng 4 trình bày tóm tắt kết quả kiểm định các giả thuyết nghiên cứu được đề xuất. Kết quả phân tích đường dẫn cung cấp bằng chứng rằng tất cả các giả thuyết $\mathrm{H} 1, \mathrm{H} 2, \mathrm{H} 3, \mathrm{H} 7$ và $\mathrm{H} 8$ đều được chấp nhận ở mức ý nghĩa thống kê $\mathrm{p}<0.001$; giả thuyết $\mathrm{H} 4$ và $\mathrm{H} 5$ được hỗ trợ ở mức ý nghĩa $\mathrm{p}<0.05$ và giả thuyết H6 cũng được chấp nhận với $\mathrm{p}=0.01$.

\section{Bảng 4}

Kết quả kiểm định giả thuyết nghiên cứu

\begin{tabular}{|c|c|c|c|c|c|}
\hline \multicolumn{2}{|r|}{ Giả thuyết } & Kỳ vọng & $\begin{array}{c}\text { Troung số } \\
\text { chuẩn hóa }\end{array}$ & P-value & Kết quả \\
\hline $\mathrm{H} 1$ & $\begin{array}{l}\text { Niềm tin có tác động tích cực } \\
\text { đến chia sẻ tri thức hiện }\end{array}$ & + & 0.522 & $\begin{array}{c}* * * \\
(\mathrm{p}<0.001)\end{array}$ & Chấp nhận \\
\hline $\mathrm{H} 2$ & $\begin{array}{l}\text { Niềm tin có tác động tích cực } \\
\text { đến chia sẻ tri thức ẩn }\end{array}$ & + & 0.568 & $\begin{array}{c}* * * \\
(\mathrm{p}<0.001)\end{array}$ & Chấp nhận \\
\hline $\mathrm{H} 3$ & $\begin{array}{l}\text { Phần thưởng của tổ chức có } \\
\text { tác động tích cực đến chia sẻ } \\
\text { tri thức hiện }\end{array}$ & + & 0.194 & $\begin{array}{c}0.001 \\
(\mathrm{p}=0.001)\end{array}$ & Chấp nhận \\
\hline $\mathrm{H} 4$ & $\begin{array}{l}\text { Phần thưởng của tổ chức có } \\
\text { tác động tích cực đến chia sẻ } \\
\text { tri thức ẩn }\end{array}$ & + & 0.118 & $\begin{array}{c}0.041 \\
(p<0.05)\end{array}$ & Chấp nhận \\
\hline H5 & $\begin{array}{l}\text { Sự hỗ trợ của quản lý có tác } \\
\text { động tích cực đến chia sẻ tri } \\
\text { thức hiện }\end{array}$ & + & 0.143 & $\begin{array}{c}0.024 \\
(\mathrm{p}<0.05)\end{array}$ & Chấp nhận \\
\hline H6 & $\begin{array}{l}\text { Sự hỗ trợ của quản lý có tác } \\
\text { động tích cực đến chia sẻ tri } \\
\text { thức ẩn }\end{array}$ & + & 0.159 & $\begin{array}{c}0.010 \\
(p=0.01)\end{array}$ & Chấp nhận \\
\hline $\mathrm{H} 7$ & $\begin{array}{l}\text { Chia sẻ tri thức hiện có ảnh } \\
\text { hươong tích cực đển hành vi } \\
\text { làm việc đổi mới }\end{array}$ & + & 0.287 & $\begin{array}{c}* * * \\
(\mathrm{p}<0.001)\end{array}$ & Chấp nhận \\
\hline $\mathrm{H} 8$ & $\begin{array}{l}\text { Chia sẻ tri thức ẩn có ảnh } \\
\text { hưởng tích cực đến hành vi } \\
\text { làm việc đổi mới }\end{array}$ & + & 0.291 & $\begin{array}{c}* * * \\
(\mathrm{p}<0.001)\end{array}$ & Chấp nhận \\
\hline
\end{tabular}

Nguồn: Tác giả 


\section{Thảo luận kết quả và hàm ý quản trị}

\subsection{Thảo luận kết quả}

Tác động của chia sẻ tri thức đối với hành vi đổi mới trong công việc đã được đề cập trong lý thuyết và một số nghiên cứu đã minh chứng mối quan hệ đó trong thực tiễn. Trong bối cảnh ngành hàng không Việt Nam, bài báo này đã xác định các tiền đề của chia sẻ tri thức để thúc đẩy nhân viên làm việc đổi mới. Kết quả nghiên cứu cho thấy tất cả các giả thuyết về ảnh hưởng của 03 nhân tố niềm tin, phần thưởng của tổ chức và sự hỗ trợ quản lý đến hành vi chia sẻ tri thức hiện và tri thức ẩn đều được chấp nhận và có ý nghĩa thống kê, trong đó tác động mạnh nhất thuộc về niềm tin giữa các nhân viên. Niềm tin là nhân tố thúc đẩy chia sẻ tri thức hiện và chia sẻ tri thức ẩn. Amayah (2013), Ali và cộng sự (2019) và Javaid và cộng sự (2020) cũng phát hiện ra rằng niềm tin giữa các nhân viên sẽ làm tăng hành vi chia sẻ tri thức. Phần thưởng của tổ chức có ảnh hưởng tích cực đến chia sẻ tri thức (hiện và ẩn). Điều này chứng minh rằng sự mong đợi của cá nhân về phần thưởng có vai trò thúc đẩy chia sẻ tri thức. Bằng nghiên cứu thực nghiệm, Hau và cộng sự (2013) chứng minh phần thưởng có tác động tích cực đến chia sẻ tri thức ẩn, còn Amayah (2013), Ali và cộng sự (2019), Javaid và cộng sự (2020) cũng chỉ ra rằng phần thưởng có vai trò quan trọng trong thúc đẩy chia sẻ tri thức. Ngoài ra, chia sẻ tri thức hiện và tri thức ẩn còn được tăng cường bởi sự hỗ trợ của quản lý trong tổ chức. Phát hiện này tương đồng với Chen và Cheng (2012) và Ali và cộng sự (2019) chứng minh vai trò tinh thần của sự hỗ trợ quản lý là cần thiết để khuyến khích nhân viên chia sẻ tri thức với nhau.

Các kết quả thực nghiệm của nghiên cứu này cũng hỗ trợ cho quan hệ tích cực giữa thực hành chia sẻ tri thức hiện và tri thức ẩn đến hành vi làm việc đổi mới của nhân viên trong ngành hàng không tại Việt Nam. Kết quả của tác giả củng cố thêm bằng chứng cho nghiên cứu của Yun và Lee (2017) cho rằng khi nhân viên tích cực chia sẻ tri thức tích cực thì họ có thêm thông tin và sẵn sàng đổi mới. Hu và cộng sự (2009), Radaelli và cộng sự (2014) cũng phát hiện ra sự tương quan giữa kết quả hành vi làm việc đổi mới và chia sẻ tri thức. Còn Afsar và cộng sự (2017) đã kiểm định vai trò của chia sẻ tri thức trong việc thúc đẩy nhân viên trong ngành hàng không làm việc đồi mới.

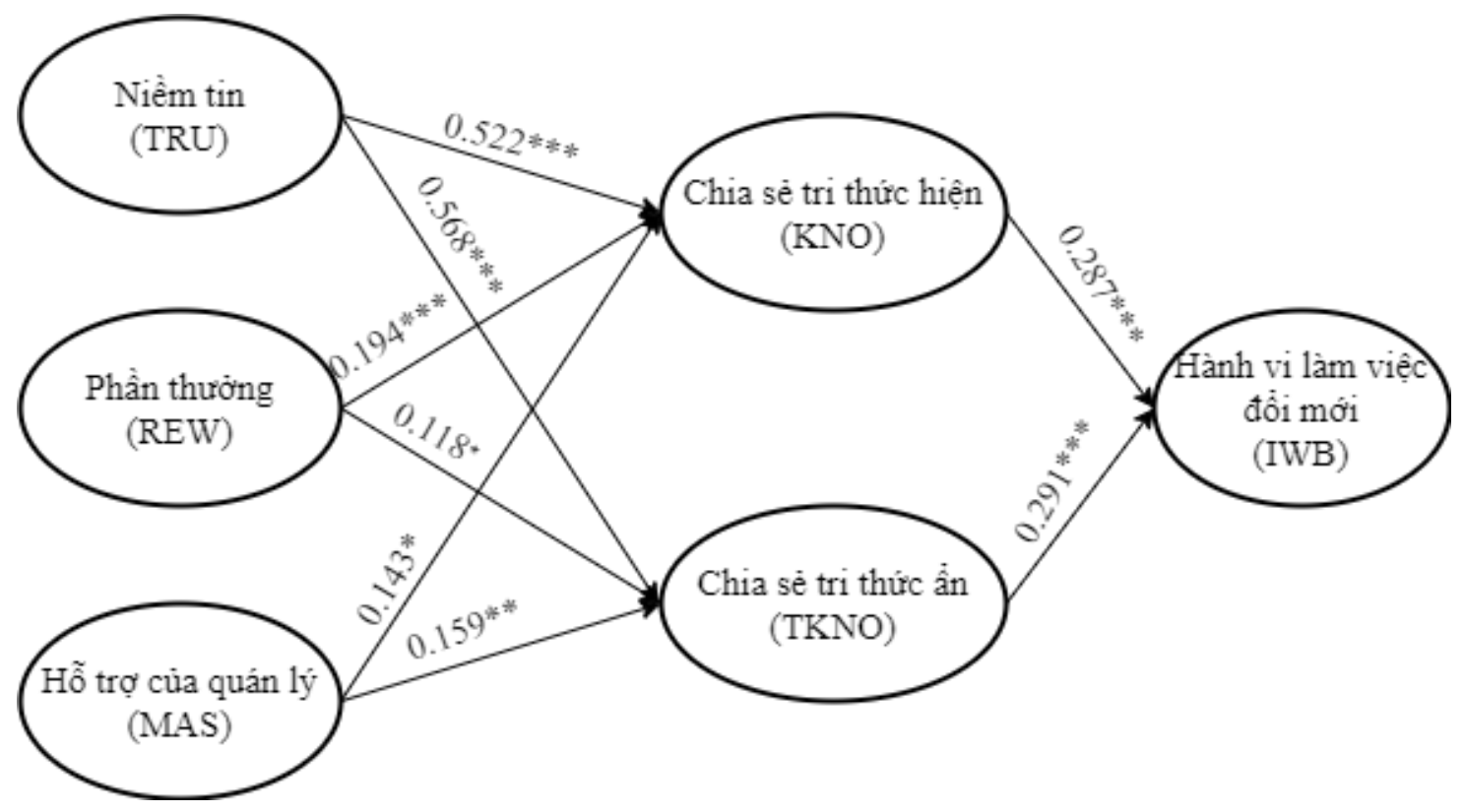

Hình 2. Mô hình kết quả nghiên cứu

Ghi chú: *: $\mathrm{p}<0.05 ; * *: \mathrm{p}<0.01 ; * * *$ p $<0.001$

Nguồn: Tác giả 


\subsection{Hàm ý quản trị}

Nghiên cứu này nhấn mạnh rằng các nhà quản lý của các công ty hàng không cần phải quan tâm đến các thực hành chia sẻ tri thức và xem nó như một công cụ quan trọng để đạt được kết quả đổi mới. Dựa vào kết quả thực nghiệm của bài báo này, tác giả đề xuất một số hàm ý để khuyến khích nhân viên chia sẻ tri thức tại nơi làm việc.

Nhân viên có xu hướng tin tưởng những đồng nghiệp có năng lực, đáng tin cậy và sẵn sàng giúp đỡ người khác. Vì vậy, để thúc đẩy niềm tin giữa các nhân viên, lãnh đạo ngành hàng không nên chủ động xây dựng văn hóa tổ chức tạo niềm tin và tăng cường sự cộng tác giữa các nhân viên bằng các chính sách và nguồn lực hợp lý. Chẳng hạn như tăng cường bầu không khí làm việc thật sự cởi mở, tạo sự công bằng, công khai, minh bạch để kiến tạo và nuôi dưỡng niềm tin nhằm tạo động lực cho việc chia sẻ tri thức và khuyến khích nhân viên luôn có những ý tưởng đổi mới, sáng tạo trong công việc.

Để khuyến khích và tăng cường chia sẻ tri thức trong toàn bộ tổ chức thì không thể thiếu sự hỗ trợ của quản lý, lãnh đạo. Người quản lý, trưởng bộ phận, người giám sát và trưởng nhóm cần thúc đẩy việc chia sẻ những ý kiến, hướng dẫn chuyên môn và thậm chí tư vấn kỹ thuật dựa trên kinh nghiệm. Người quản lý có thể khuyến khích nhân viên chia sẻ tri thức, tạo thành một phần trong thói quen hàng ngày của họ. Ngoài ra, lãnh đạo cao nhất nên hỗ trợ các hoạt động giúp tăng cường giao tiếp và trao đổi những ý tưởng và kinh nghiệm. Hơn nữa, lãnh đạo cao nhất phải tôn trọng và phản hồi các ý tưởng và mối quan tâm của nhân viên. Hỗ trợ quản lý cho phép nhân viên chia sẻ kinh nghiệm và phát triển các kỹ năng mới.

Các công ty hàng không có thể xây dựng hệ thống khen thưởng phi tiền tệ và hệ thống khen thưởng tiền tệ một cách đầy đủ, hợp lý để khuyến khích nhân viên của mình chia sẻ những kiến thức, kỹ năng, kinh nghiệm. Phần thưởng tiền tệ có thể là tăng lương và tiền thưởng cho những nhân viên có thành tích xuất sắc trong việc chia sẻ và đóng góp tri thức trong tổ chức. Phần thưởng phi tiền tệ thể hiện bằng sự công nhận, biểu dương các nhân viên có vai trò tích cực đối với việc thúc đẩy chia sẻ tri thức, chẳng hạn như tạo cơ hội ưu tiên trong đào tạo, quy hoạch, bổ nhiệm.

\section{Kết luận và hướng nghiên cứu tiếp theo}

Bối cảnh kinh doanh của các công ty hàng không ngày nay được đánh dấu bằng sự cạnh tranh gay gắt và không chắc chắn đã buộc các tổ chức phải liên tục nổ lực để thúc đẩy một môi trường đổi mới. Một trong những chiến lược chính mà một tổ chức có thể thực hiện để đạt được sự đổi mới là quản lý và chia sẻ tri thức hiệu quả. Nghiên cứu này đã phân tích vai trò của chia sẻ tri thức đối với hành vi đổi mới của nhân viên với tác động của niềm tin, phần thưởng của tổ chức và sự hỗ trợ của quản lý. Trong đó, niềm tin là động lực mạnh nhất để nhân viên chia sẻ tri thức và đổi mới, sáng tạo trong công việc.

Tác giả đã hoàn thành các mục tiêu nghiên cứu, nhưng không tránh khỏi một số hạn chế và cần phải thực hiện tiếp theo. Trước hết, có thể nghiên cứu thêm về vai trò của các nhân tố tổ chức như văn hóa tổ chức, cấu trúc tổ chức và các nhân tố động lực như niềm tin vào tri thức của bản thân, thái độ và nhận thức đối với hành vi chia sẻ tri thức. Ngoài ra, để mô hình đầy đủ hơn thì nghiên cứu tiếp theo có thể bổ sung nhân tố hiệu quả làm việc như là kết quả của hành vi làm việc đổi mới. 


\section{Tài liệu tham khảo}

Afsar, B., Safdar, U., Dost, M., \& Ali, Z. (2017). Flight attendant's knowledge sharing, innovative work behaviour, and new service development. International Journal of Work Innovation, 2(2/3), 193-215.

Akram, T., Lei, S., Haider, M. J., \& Hussain, S. T. (2018). Exploring the impact of knowledge sharing on the innovative work behavior of employees: A study in China. International Business Research, 11(3), 186-194.

Alhady, M., Idris, A., Sawal, M., Azmi, N., \& Zakaria, Z. (2011). Knowledge sharing behavior and individual factors: A relationship study in the i-Class environment. Proceeding of the International Conference on Management and Artificial Intelligence, 6(2011), 137-141.

Ali, A. A., Paris, L., \& Gunasekaran, A. (2019). Key factors influencing knowledge sharing practices and its relationship with organizational performance within the oil and gas industry. Journal of Knowledge Management, 23(9), 1806-1837.

Amayah, A. T. (2013). Determinants of knowledge sharing in a public sector organization. Journal of Knowledge Management, 17(3), 454-471.

Anantatmula, V. S. (2008). Leadership role in making effective use of KM. The Journal of Information and Knowledge Management Systems, 38(4), 445-460.

Anderson, J. C., \& Gerbing, D. W. (1988). Structural equation modeling in practice: A review and recommended two-step approach. Psychological Bulletin, 103(3), 411-423.

Barney, J. (1991). Firm resources and sustained competitive advantage. Journal of Management, 17(1), 99-120.

Bock, G. W., \& Kim, Y. G. (2002). Breaking the myths of rewards: An exploratory study of attitudes about knowledge sharing. Information Resources Management Journal, 15(2), 14-21.

Bock, G. W., Zmud, R. W., Kim, Y. G., \& Lee, J. N. (2005). Behavioral intention formation in knowledge sharing: Examining the roles of extrinsic motivators, social-psychological forces, and organizational climate. MIS Quarterly, 29(1), 87-111.

Cabrera, A., Collins, W. C., \& Salgado, J. F. (2006). Determinants of individual engagement in knowledge sharing. The International Journal of Human Resource Management, 17(2), 245-264.

Chen, W. J., \& Cheng, H. Y. (2012). Factors affecting the knowledge sharing attitude of hotel service personnel. International Journal of Hospitality Management, 31(2), 468-476.

Cheng, M. Y., Ho, J. S. Y., \& Lau, P. M. (2009). Knowledge sharing in academic institutions: A study of multimedia university Malaysia. Electronic Journal of Knowledge Management, 7(3), 313-324.

Chow, W. S., \& Chan, L. S. (2008). Social network, social trust and shared goals in organizational knowledge sharing. Information \& Management, 45(7), 458-465.

Foos, T., Schum, G., \& Rothenberg, S. (2006). Tacit knowledge transfer and the knowledge disconnect. Journal of Knowledge Management, 10(1), 6-18.

Grant, R. M. (1996). Toward a knowledge- based theory of the firm. Strategic Management Journal, 17(S2), 109-122. 
Hair, J. F., Black, W. C., Babin, B. J., \& Anderson, R. E. (2014). Multivariate data analysis. London, UK: Pearson.

Hau, Y. S., Kim, B., Lee, H., \& Kim, Y. G. (2013). The effects of individual motivations and social capital on employees' tacit and explicit knowledge sharing intentions. International Journal of Information Management, 33(2), 356-366.

Hsu, I. C., \& Sabherwal, R. (2012). Relationship between intellectual capital and knowledge management: An empirical investigation. Decision Sciences, 43(3), 489-524.

Hsu, M. H., Ju, T. L., Yen, C. H., \& Chang, C. M. (2007). Knowledge sharing behavior in virtual communities: The relationship between trust, self-efficacy, and outcome expectations. International Journal of Human-Computer Studies, 65(2), 153-169.

Hu, M. L. M., Horng, J. S., \& Sun, Y. H. C. (2009). Hospitality teams: Knowledge sharing and service innovation performance. Tourism Management, 30(1), 41-50.

Huang, Q., Davison, R. M., \& Gu, J. (2011). The impact of trust, guanxi orientation and face on the intention of Chinese employees and managers to engage in peer- to- peer tacit and explicit knowledge sharing. Information Systems Journal, 21(6), 557-577.

Inkpen, A. C., \& Tsang, E. W. (2005). Social capital, networks, and knowledge transfer. Academy of Management Review, 30(1), 146-165.

Janssen, O. (2000). Job demands, perceptions of effort-reward fairness and innovative work behaviour. Journal of Occupational and Organizational Psychology, 73(3), 287-302.

Javaid, J., Soroya, S., \& Mahmood, K. (2020). Impact of personal and organizational factors on knowledge sharing attitude of university teachers in Pakistan. The Electronic Library, 38(2), 317-336.

Kamaşak, R., \& Bulutlar, F. (2010). The influence of knowledge sharing on innovation. European Business Review, 22(3), 306-317.

Kline, R. B. (2015). Principles and practice of structural equation modeling. New York, NY: Guilford.

Lee, J. C., Shiue, Y. C., \& Chen, C. Y. (2016). Examining the impacts of organizational culture and top management support of knowledge sharing on the success of software process improvement. Computers in Human Behavior, 54(1), 462-474.

Lee, K. J. (2016). Sense of calling and career satisfaction of hotel frontline employees: Mediation through knowledge sharing with organizational members. International Journal of Contemporary Hospitality Management, 28(2), 346-365.

Liu, Y., \& Phillips, J. S. (2011). Examining the antecedents of knowledge sharing in facilitating team innovativeness from a multilevel perspective. International Journal of Information Management, 31(1), 44-52.

Mishra, M., \& Pandey, A. (2019). The impact of leadership styles on knowledge-sharing behavior: A review of literature. Development and Learning in Organizations: An International Journal, 33(1), 16-19.

Nonaka, I. (1994). A dynamic theory of organizational knowledge creation. Organization Science, 5(1), 14-37. 
Radaelli, G., Lettieri, E., Mura, M., \& Spiller, N. (2014). Knowledge sharing and innovative work behaviour in healthcare: A micro-level investigation of direct and indirect effects. Creativity and Innovation Management, 23(4), 400-414.

Raykov, M. (2014). Employer support for innovative work and employees' job satisfaction and job-related stress. Journal of Occupational Health, 56(4), 244-251.

Reychav, I., \& Weisberg, J. (2010). Bridging intention and behavior of knowledge sharing. Journal of Knowledge Management, 14(2), 285-300.

Tsai, W., \& Ghoshal, S. (1998). Social capital and value creation: The role of intrafirm networks. The Academy of Management Journal, 41(4), 464-476.

Wang, S., \& Noe, R. A. (2010). Knowledge sharing: A review and directions for future research. Human Resource Management Review, 20(2), 115-131.

Wang, Z., \& Wang, N. (2012). Knowledge sharing, innovation and firm performance. Expert Systems with Applications, 39(10), 8899-8908.

Wang, Z., Wang, N., \& Liang, H. (2014). Knowledge sharing, intellectual capital and firm performance. Management Decision, 52(2), 230-258.

Yun, Y. J., \& Lee, K. J. (2017). Social skills as a moderator between R\&D personnel's knowledge sharing and job performance. Journal of Managerial Psychology, 32(5), 387-400.

Zaqout, F., \& Abbas, M. (2012). Towards a model for understanding the influence of the factors that stimulate university students' engagement and performance in knowledge sharing. Library Review, 61(5), 345-361.

Zhou, K. Z., \& Li, C. B. (2012). How knowledge affects radical innovation: Knowledge base, market knowledge acquisition, and internal knowledge sharing. Strategic Management Journal, 33(9), 1090-1102. 